\title{
Developing a web based interactive 3D virtual environment for novel skin measurement instruments
}

\author{
Omar AlHashimi ${ }^{*} 1$, Perry Xiao ${ }^{* 2}$ \\ * College of Applied Sciences - Sohar \\ Sohar province, P O Box 135, Postal Code 311, Oman \\ ${ }^{1}$ omar 108@hotmail.com or ${ }^{1}$ omar. soh@cas.edu.om \\ * London South Bank University \\ 103 Borough Road, London SE1 0AA, UK \\ 2 xiaop@ lsbu.ac.uk
}

The advent and the wide use of 3D online contents, 3D

\begin{abstract}
D design and 3D contents are becoming ever so popular in today's technology. The whole world is moving towards the 3D content platform, 3D printers, 3D TV sets, $3 \mathrm{D}$ mobiles etc. no exception to that is the web and all things in it.

AquaFlux and Epsilon are two of our state of the art skin measurement instruments. Originally developed by our research group as research projects, AquaFlux and Epsilon have now been commercialised and used in more than 70 organis ations worldwide. However, due to the nature of the technologies, they often require on-site intensive training, which is expensive and time-consuming. There is a genuine need for an interactive virtual training environment that users can be trained whenever and wherever they want. This paper demonstrates the development of a web-based interactive 3D virtual environment for these two skin measurement instruments to serve this demand. The new system contains detailed virtual user manuals, step by step interactive instructions that will direct the users on how to use these two skin measurement devices. The virtual environment will benefit us ers by giving users a very realis tic feel about how these instruments work. VR (Virtual Reality) is an essential technology that will deliver to users the experience and knowledge in this regard.
\end{abstract}

Keywords-3D modelling, Web3D, Virtual Reality (VR), LMS (Learning Management System), E-Learning, Web 3D Applications, AquaFlux, Epsilon, 3ds Max.

\section{INTRODUCTION}

This research paper illustrates how the technology of VR is vital in conveying a certain notion to any audience or users through a created realistic environment. The process of demonstration of any newly purchased device is another essential aspect to utilise all the functions of that device or instrument. Virtualization or the virtual world has always been connected to $3 \mathrm{D}$, as it is the best way to demonstrate any $3 \mathrm{D}$ object, picture, movie etc. The term virtualisation broadly describes the separation of a resource or request for a service from the underlying physical delivery of that service. An important addition to make virtualisation more usable and beneficial is the interactive 3D multimedia applications that can be used in it especially when the process presented online via the Internet [1]. Also, users are well prepared for the shift from $2 \mathrm{D}$ to $3 \mathrm{D}$ interfaces.
Games and 3D movies on a fast operating system, big RAM size, and great display capabilities have increased the need to adopt new 3D technologies and produce a very sophisticated and realistic product. And now is the time to develop further and enhance the utilisation of those 3D technologies and interactive environments to other areas, such as e-commerce, museums, e-learning, online training, LMS, tourism, and the medical sector. Bringing in those 3D interactive tools and environments into these new areas has raised and improved the user experience immensely and made it more exciting. Online 3D virtual environments (second life) have increased users' concerns. They represent real-world features and characteristics to clients and allow them to interact with it via using the internet as a platform. 3D virtual environment accessed remotely online would be as feasible as accessing any application locally. Accordingly, the notion of VR can be integrated with social media to further advance its appeal. However, a recent VMware report states that "the potential of 3D/VR technology in everyday applications can be fully exploited. Only if accompanied by the development of efficient and easy to use methods of creation, management, search and presentation of interactive 3D multimedia content, which could be used by both expert and non-expert users" [2]. In this regard, London South Bank University eng ineer ing lab has built and developed AquaFlux and Epsilon, which are medical devices. Demonstrating these medical instruments in VR environments would illustrate their functionality as used in the real world. Xu (2015) describes AquaFlux as:

a novel condenser based, closed chamber technology for measuring water vapour flux density from arbitrary surfaces, including in-vivo measurements of transepidermal water loss (TEWL), skin surface water loss (SSWL) and perspiration. It uses a cylindrical measurement chamber, with one end open and attached to the sample surface, and another end closed and cooled down to below freezing point [3].

Comparing with other technologies, AquaFlux has higher sensitivity, higher repeatability, and above all, the measurement results are independent of external environment. Biox (2014) describes Epsilon as:

a novel instrument for imaging dielectric permittivity $(\varepsilon)$ of a wide variety of soft materials, including animal and plant tissues, waxes, fats, gels, liquids, and 
powders. Its proprietary electronics and signal processing transform the sensor's native non-linear signals into a calibrated permittivity scale for imaging properties such as hydration or recording dynamic processes such as textile wetting or the permeation of liquids through membranes [13].

The Epsilon user manual states that "The system consists of a hand-held probe, a parking base, and an in-vitro stand, securely stored in a purpose-designed case" [4]. The two instruments are shown in the figure below (Fig. 1).
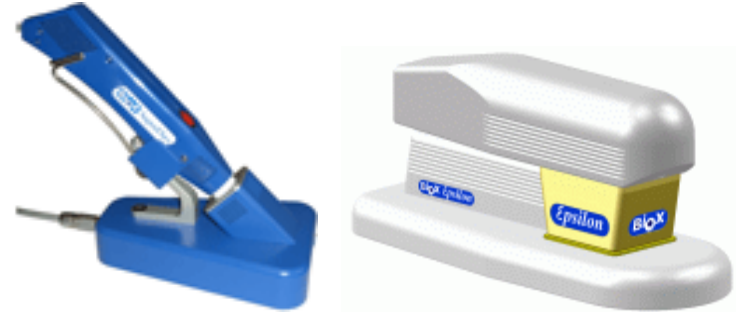

Fig. 1. AquaFlux (Left), Epsilon (Right)

\section{USAGES OF 3D CONTENTS AND RELATED WORK}

Since the arrival of Web 2, there has been a significant development in web applications. Web II aids and covers diverse activities, such as teamwork, communication, social networking, and involvement between computer and Internet users. The three-dimensional immersive virtual worlds (3DVW) are one of the essential applications of Web II, which are computer-generated, virtual, online, graphics, multimedia and threedimensional environments [5]. 3D contents and 3D objects have been around the world of technology since the need for animation and animated content to be used in computer systems during the era of the 1970s in computer graphics. The main idea of creating such an object in three dimensions is to show users the reality of the object. 3D modelling is the most important and essential work in the field of virtual reality technology [9]. The process of creating a $3 \mathrm{D}$ object can be divided into three stages:

1- 3D modelling: the process of building a computer model in 3 dimensions.

2- Layout and animation: the process of adding animation and creating a scene to the object.

3- Rendering: the process of adding other effects to our scenes, such as lights, surface type, positioning the camera and other qualities.

$3 \mathrm{D}$ contents are used a lot in the area of science, technology, engineering, medical environment, tourism, cosmetic, simulation, e-learning and many more. It has been used in the disciplines mentioned above combined with a virtual presentation to stimulate the presented content as is used and tested in the real world. Some emerging technologies will overcome some of the difficulties in those areas. These include computer graphics, augmented reality, computational dynamics and virtual worlds.
Recently we have seen some new ideas appearing in the literature concerned with the future of education. As has been pointed out by Potkonjak, Gardner, Callaghan, Mattila, Guet1, Petrović, Jovanović (2016), "Technological examples most relevant are distance learning, e-learning, virtual laboratories, virtual reality and virtual worlds, avatars. Dynamics-based virtual systems, and the overall new concept of immersive education that integrates many of these ideas together" [6]. The ARCO project (Augmented Representation of Cultural Objects) directs at building up the whole set of technologies to help museums to produce, change, control and deliver digitised cultural objects in virtual exhibitions reachable from within as well as from outside the museums. Wojciechowski, Walczak, White, Cellary (2004) state that:

The ARCO system consists of three main architectural components: content production, content management, and visualisation. The content production includes all tools and techniques used to create digital representations of museum artefacts. The method of modelling depends on features of the objects. Objects with simple geometry can be modelled with a state-ofthe-art modelling package such as $3 d s$ max equipped with a set of additional plug-ins developed in the ARCO project. The plugins simplify the process of creating virtual models of cultural objects by providing a user with functionality tailored to perform particular modelling tasks. Digital representations are stored in the ARCO database and managed with ARCO Content Management Application (ACMA). The visualisation of the digital representations of museum artefacts is performed by Virtual and Augmented Reality interfaces. The interfaces combine a Web-based form of presentation with either VR or AR virtual exhibitions. The Web-based form of presentation allows users to search and browse the database contents by the use of a well-known interface, whereas the $\mathrm{VR}$ and $\mathrm{AR}$ exhibitions let them examine virtual reconstructions of selected objects in 3D environments. The virtual exhibitions displayed in the end-user interfaces are dynamically generated based on parameterised visualis ation templates and the database contents [7].

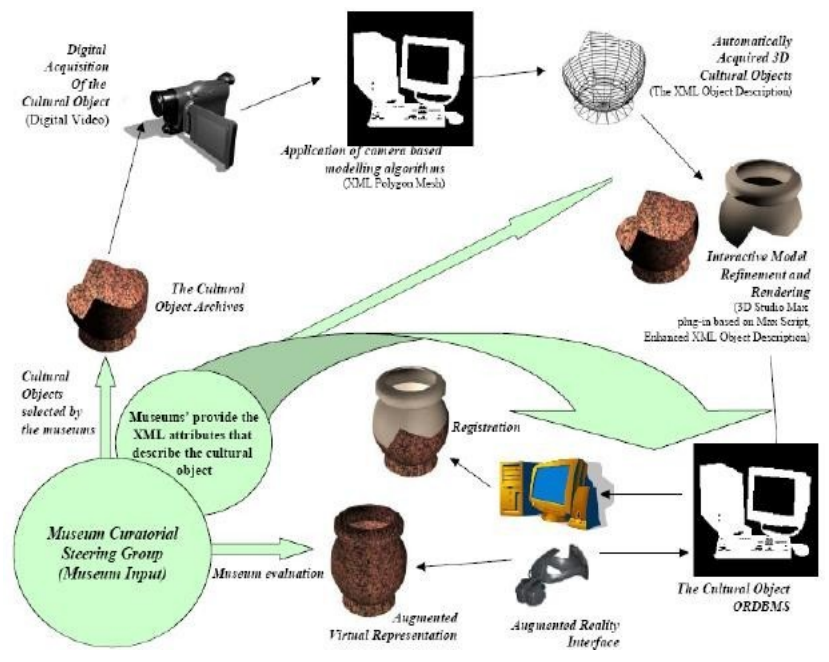

Fig. 2. The overall architecture of ARCO [7] 


\section{DESIGN, DEVELOP AND IMPLEMENT OF AQUAFLUX AND EPSILON VUM (VIRTUAL USER MANUAL)}

The design process was a little complicated and faced different options for choosing the right 3D modelling package, in this project the software modelling package used is 3ds Max. And to add interactivity between the user (client) and the instruments the software Adobe Flash CS6 is used. Other modelling packages were used at the early stages of this project such as Google Sketchup, Blender and Unity but as a final result of the research was to create, build and develop the objects in 3ds Max for its professionalism and a comprehensive set of options features and functions AquaFlux and Epsilon are two medical instruments used for skin treatment, and they belong to the health and medical sector.

The Medical area is hugely associated with the use of advanced technologies such as 3D and VR for illustration, training, testing and conducting several medical procedures. "3DVWs (3D Virtual Worlds) have been used in various applications in health-related activities, and we identified them in five main categories: education; treatment; simulation; evaluation; lifestyle [5].

\section{A. Designing and Developing AquaFlux and Epsilon using 3ds Max modelling package}

$3 \mathrm{ds}$ Max, the software which can quickly and efficiently generate 3D modelling [8]. As in any 3D modelling software we have to model the instruments, AquaFlux and Epsilon contains a base part and a probe, on the base part there are few modelling challenges such as cabling input port and buttons that we need to switch left or right in AquaFlux case. The probe and the base have been measured with a real ruler to allow us to design the objects in a very realist shape as well as we should consider some photos or references to capture the right proportion for each object, e.g. how big will be the probe compared to a human hand. After the designer evaluates the size and proportion of each object, we use 3D Studio Max to model the two instruments, modelling tools are shown in the figures below for AquaFlux and Epsilon:

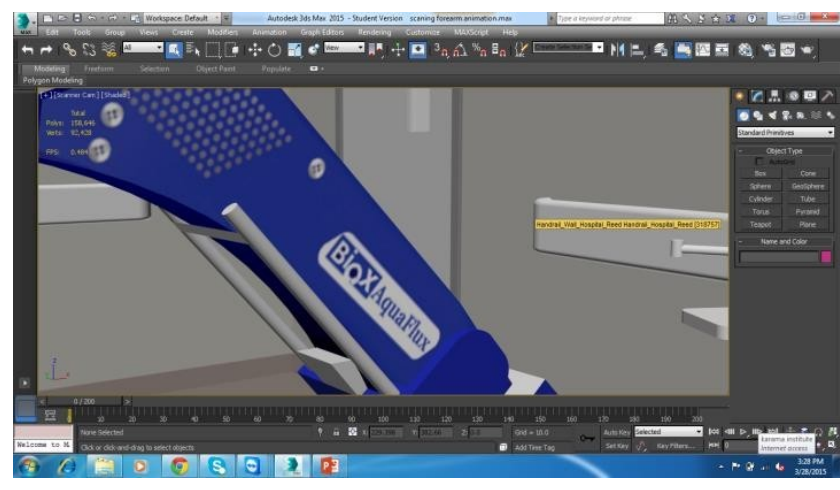

Fig. 3. The modelling of AquaFlux probe in 3 ds Max

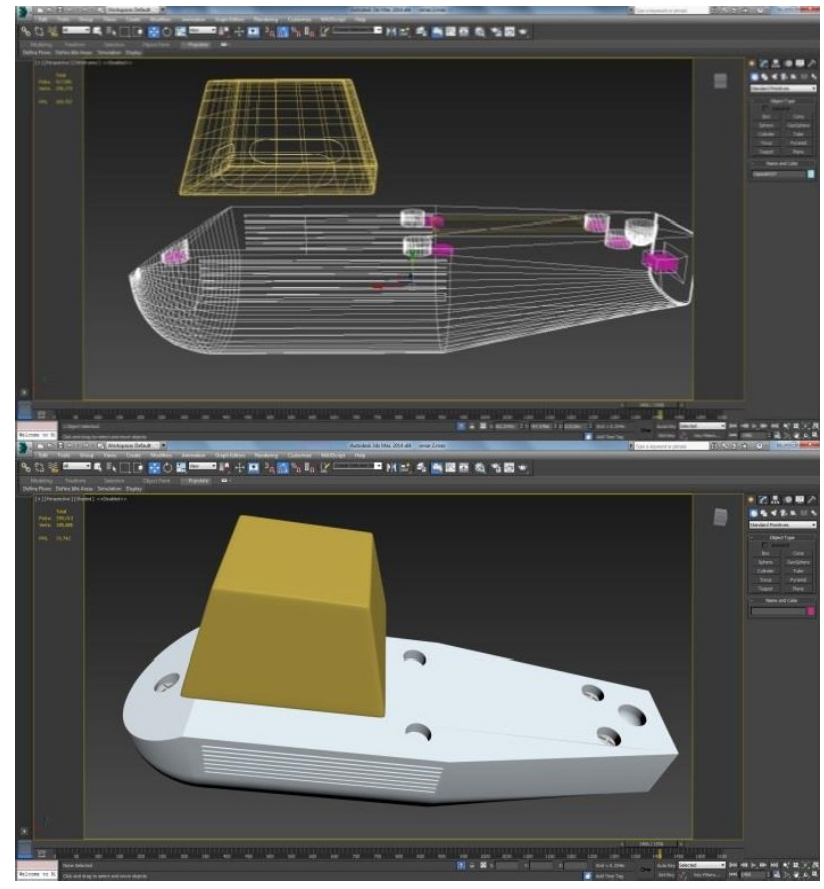

Fig. 4. The modelling of Epsilon probe in $3 \mathrm{ds}$ Max

We can insert different object types from the modelling menu such as a Box, Cone, and Sphere, Tube and Cylinder and naming each part. As the process of modelling is going along, the designer needs to make an actual reference to how the newly modelled object is very close to its realistic original copy by placing the original object picture near the modelled one as shown below:

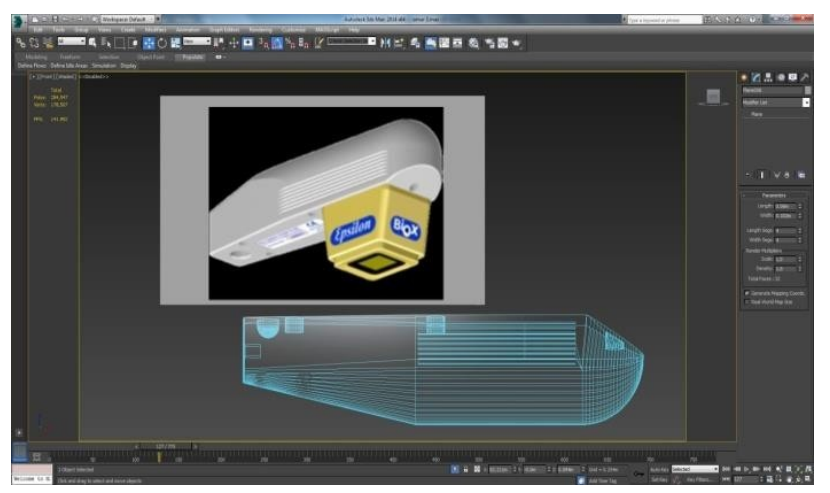

Fig. 5. Epsilon original picture compared to an Epsilon object.

\section{B. Adding materials to the AquaFlux and Epsilon instruments}

Materials should be planned to suit the object reference if it's realistic enough to meet the client's need. The choice of the material is then carefully studied so that it will not render too slow and too unrealistic. Materials with high glossiness and reflection will take so much of the rendering time, but without it, the object would be so unrealistic to users. Vray materials are used in this project, and our thirdparty renderer is vray for the whole project, it is one of the industry standards for producing professional images. 
For the Epsilon Probe and other objects, a semi-gloss material with a whitish material is used. With 0.6 glossiness and a subdivision of 20 , so that it will not take so much of the rendering time for this object because it is one of the prominent parts of this project. We used HDRI (High Definition Range Imaging) for the background to make it a little bit realistic for the lights and shadow. HDRI is useful to imitate the real world shadows and lighting that will be projected on the objects. Although HDRI will take more time in rendering, the glossiness and reflectivity of the object are turned a little bit low, and that will compensate on the runtime.

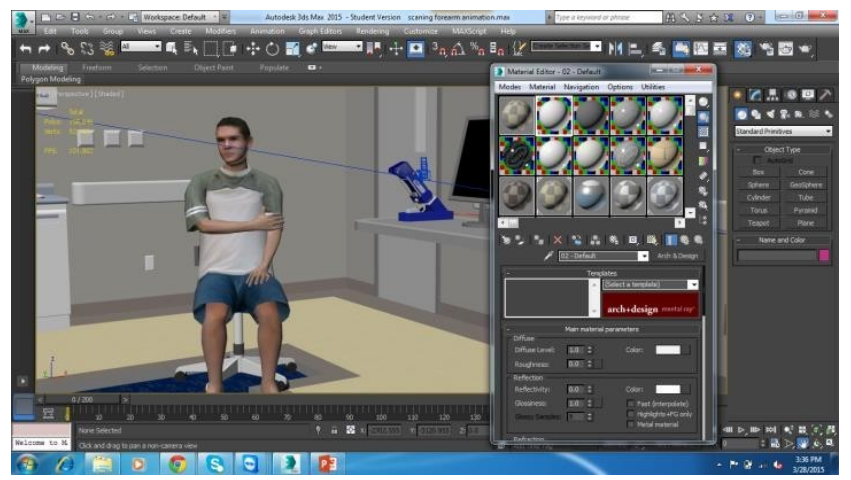

Fig. 6. Materials added to AquaFlux using the material menu

After the materials have been finally decided and added considering the time and quality of the project, we prepare the objects to be animated. It means that all objects that can be grouped will be grouped and linked with each other because if any object that has not been grouped and left out, the keyframes related to that object will be gone after this and the object will no longer be animated.

\section{Animating AquaFlux and Epsilon instruments}

Animation is a very important and crucial step in our project design. Nearly a third of the time of our work will be spent on animating the objects modelled. We need to plan earlier the scenario that each object in our scene how will interact with the other objects, the motion and the direction of movement that the specified object will go through in our virtual world. It's a quite time-consuming stage. Unlike character animation, this kind of animation is a bit simpler because it does not need bones and other complex rigging tools. The Pivotal points where all motions begin should also be considered before starting the animation. During the animation period, the Timeline is set to 1000 frames and 15 frames per second because this project is dedicated to being used in an online environment. The frames per second are lowered to accommodate the file size of the images that will be rendered for online purposes. Using the curve editor to animate objects, by editing the curve points the animated objects can be controlled (speed, timing, and movements).

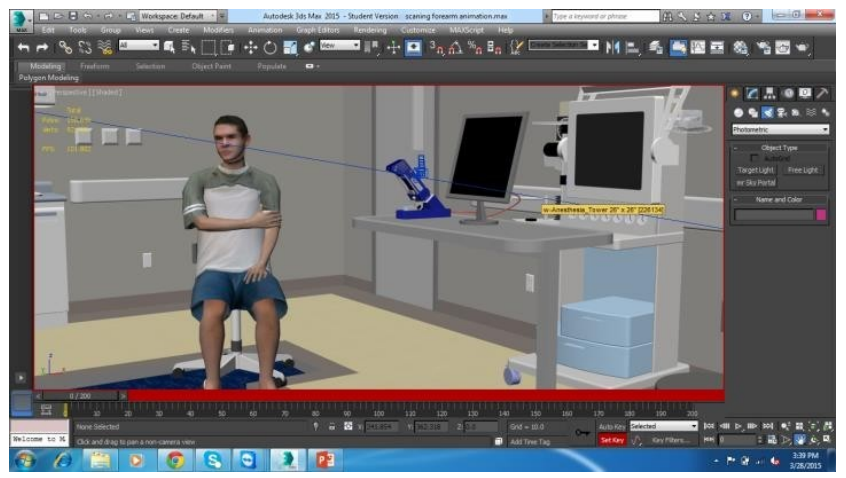

Fig. 7. Adding keyframes in 3 ds Max

\section{Rendering AquaFlux and Epsilon instruments}

After we are done with animation and setting keyframes from a certain point or number into where we want our object to stop at a specific number, then our objects are ready for the final stage of the modelling process which is rendering, where the designer can relax and watch the frames that are being rendered. However, at this stage of the work, it's very important to observe how fast and how long each object will take to finish. Each frame is carefully examined if it's in the right place (number), the objects have the right shadows and lighting. Sometimes objects can be seen floating that is not noticed during the modelling and animating phase. During Rendering, a small window will appear, rendering every square pixel calculating the objects materials, shadows, light reflections etc. In the rendering menu box we can choose the rendered images format, and the images file location, so we can export them into Flash to do all the linking afterwards.

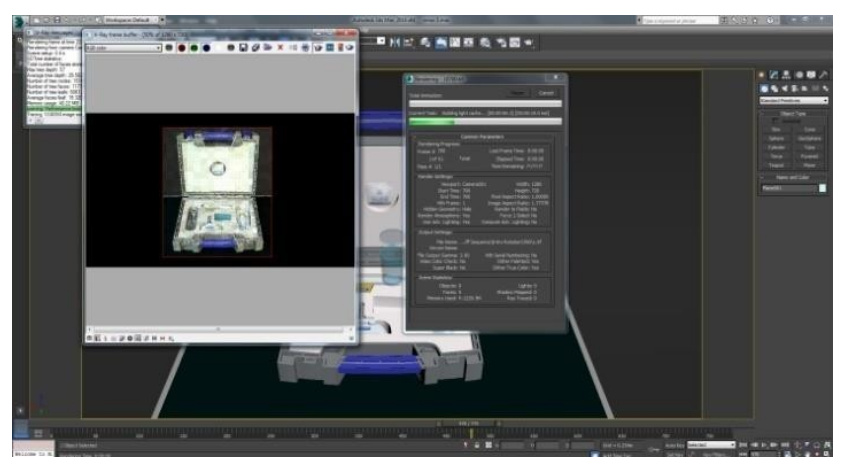

Fig. 8. Rendering window after all keyframes and file setup completed

\section{E. Adding links and Interactivity to the instruments using} Flash CS6:

To create or to add interactivity to both instruments AquaFlux and Epsilon, we needed to import all image sequences that we rendered previously using $3 \mathrm{ds}$ Max software into a new Flash:

1) Open flash file with the dimensions of $1920 \times 1080 \mathrm{HD}$ in scene 1, every one collection of motions can be put together into a scene and enable them to interact to achieve its task. 
2) Import images sequence animation into the file library.

3) Create a new symbol and start to drag images into that symbol frame by frame.

4) The image sequence that has been exported to Flash from 3ds Max is being placed on the frames in Flash, each one on a certain frame point to create something similar to the old method of cartoon flip book.

5) When we want to create a button that will stop or end action from occurring, then we only add an ActionScript that contains the word stop(); and attach it to the newly created button.

6) There are many built-in functions for ActionScript already included in a flash under global functions submenu such as movie clip control functions, timeline control, browse and printing control.

7) For example, under timeline control, we will find common functions such as (play, stop and goto).

8) If any created button that contains a script attached to it needed to be used with its functionality in a different location, then we can only create a new layer in a new scene and copy and paste that button into the new layer, and the new layer will have the exact function as the original button. As well as adding Interactivity functions and buttons to instruments, voice clips and audio been added that will play along while displaying the illustrative text once been clicked on by the user.

Above steps can be repeated to all objects that needed to be linked and interacted with the user in a virtual environment via buttons in Flash.

AquaFlux interactive user manual is a fully interactive website that can show and demonstrate all functions, features and a sample skin measurement taken from a patient. The below figures show some of the steps of how to use the Virtual User Manual.

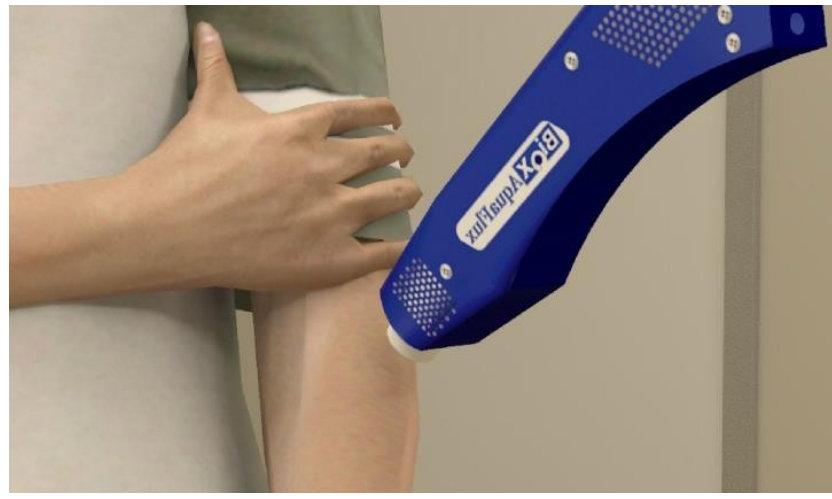

Fig. 9 AquaFlux probe is moving out of the parking stand to conduct a measurement of a patient

The user can click at the AquaFlux probe to instruct the probe to move into a patient's forearm and conduct a measurement, every step of this virtual user manual is aided with audio instructions recorder previously to help to illustrate the operational procedure of these two instruments and how it's been done.

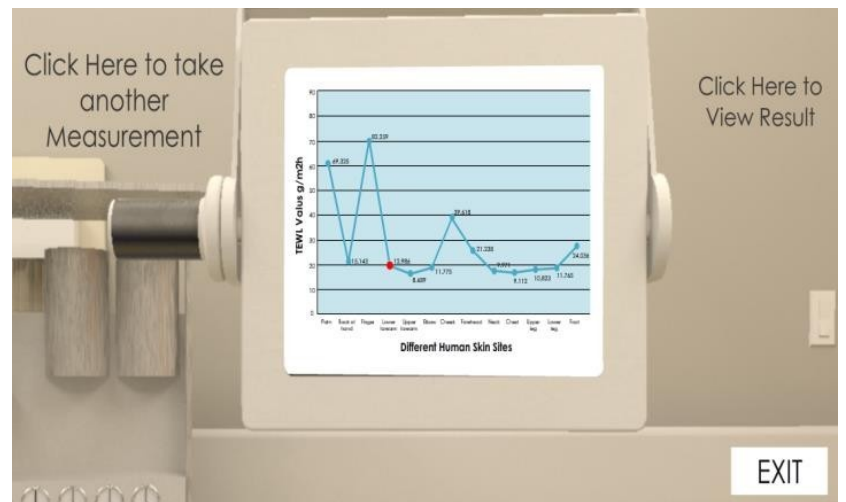

Fig. 10. Skin measurement reading will be shown on a monit or

The user can click at any item in the virtual user manual's drop-down menu, resulting in displaying a popup window to the right explaining or mentioning information about the AquaFlux's probe with little details written in the footer of the page as shown in (Fig. 12). Users also can repeat the step by clicking on the repeat button (bottom right). All other options of the VUM menu will be displayed in the same way in similar steps, all components of the AquaFlux will be shown in a 3D environment.

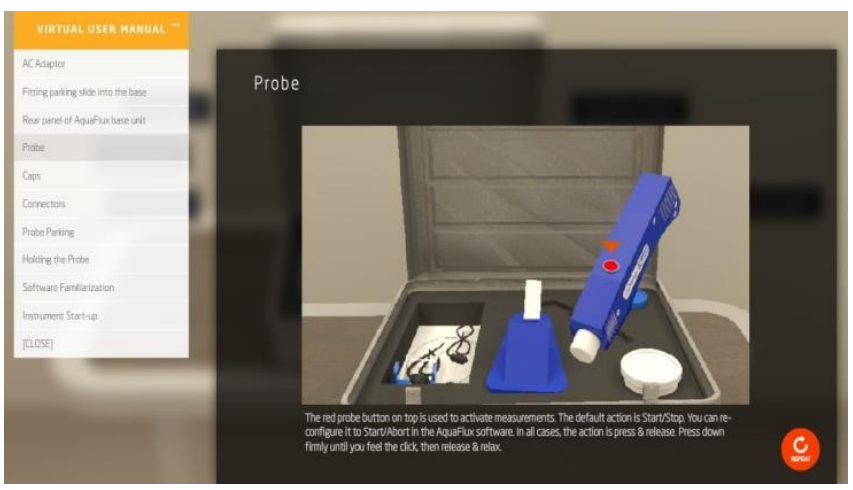

Fig. 11. AquaFlux VUM, audio aid will support each step to illustrate it further

Epsilon VUM's following a very similar way of conducting a skin measurement process for medical purposes. As previously showed some figures for AquaFlux in operation, in (Fig. 13) we can see Epsilon device in operation.

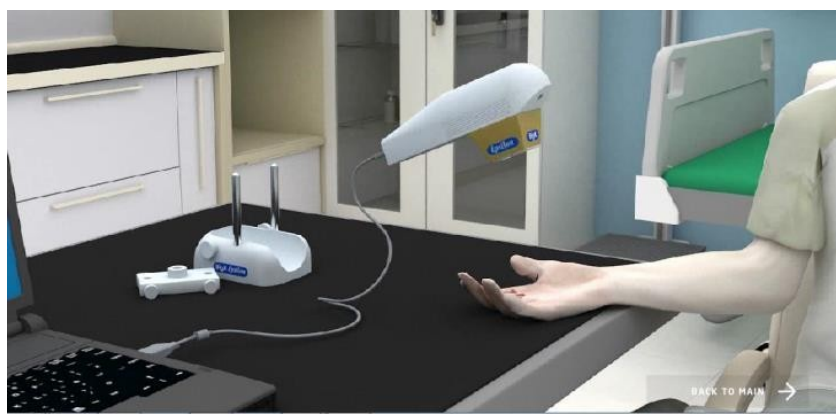

Fig. 12. Epsilon conducting a skin measurement on a patient 
After completing the measurement, the camera will focus on the laptop monitor to show the scan results as shown in (Fig. 13).

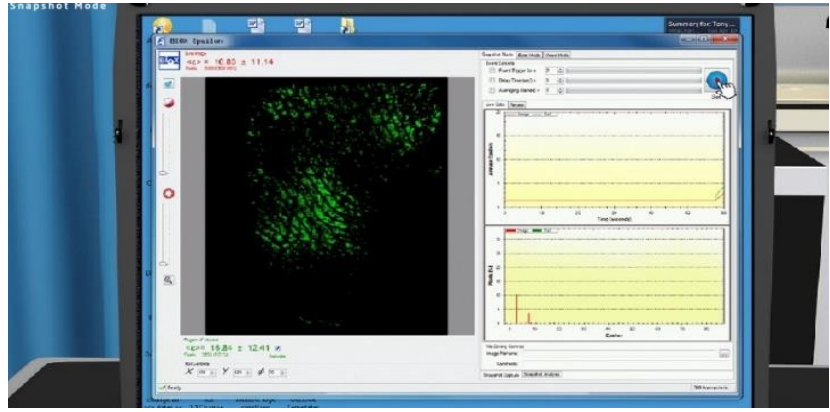

Fig. 13. Measurement result is shown above after clicking on the blue button, top right corner.

In Epsilon device, the user can conduct skin measurements in three different modes, snapshot, burst and video modes. All images and videos of scan results are saved in a folder on the machine hard drive. All buttons and tabs are designed in an interactive 3D environment and clickable by the user.

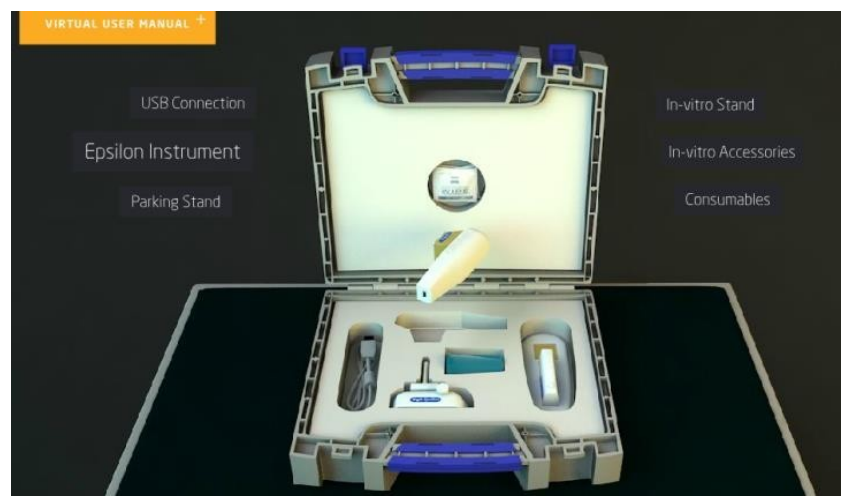

Fig. 14. Epsilon protective case and its parts displayed in a 3D interactive environment

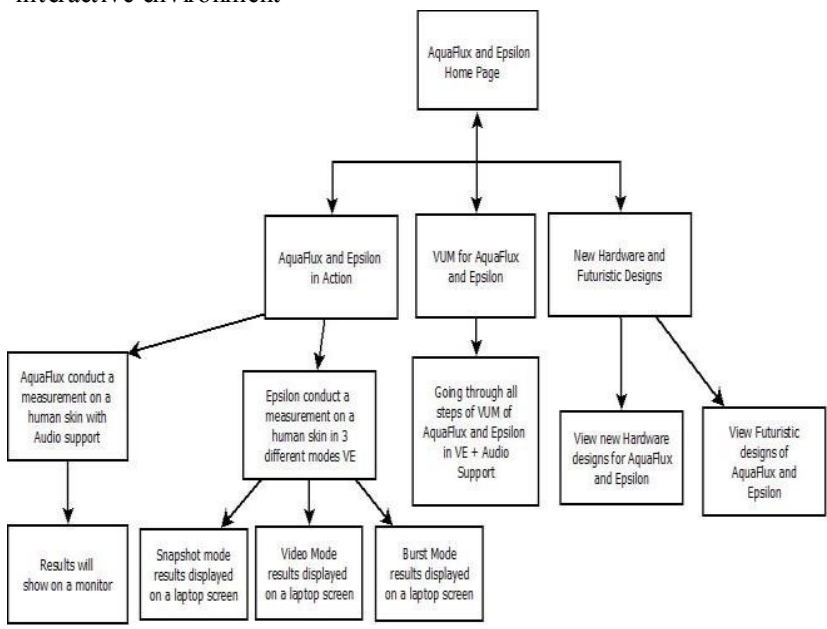

Fig. 15. Flow chart of AquaFlux and Epsilon 3D Virtual Environment

\section{NEW HARDWARE AND HOLDER DESIGN OF AQUAFLUX AND EPSILON}

By the later stages of this research, a new idea came to light, and it has a great benefit to the whole project. Also, it will make a great addition to it. The idea was to design and create a new accessory (holder) for both instruments, in which going to add a huge advantage to the measuring process to make it more quick, efficient and allow to measure multiple people (patients) at the same time. The holders created have been approved by the BIOX lab at London South Bank University. The new accessory for Epsilon (holder) was carefully planned and meticulously designed to accommodate the epsilons structure and patient's comfort. The material added to the new accessory was a cloth looking material to imitate the real world object.

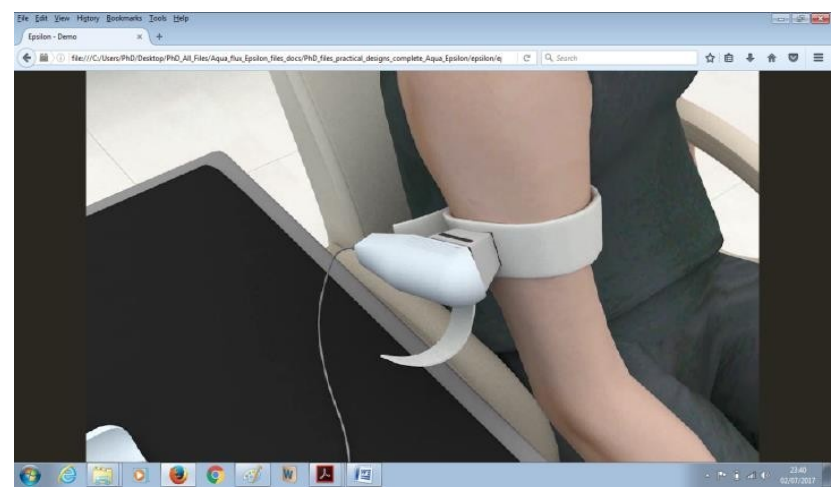

Fig. 16. Epsilon newly designed holder strap in action

Regarding Aquaflux, it was a bit trickier to design the new accessory because of the size and shape of the AquaFlux probe, but after carefully studying the structure and thoughtful planning, the holder creation was completed and the newly designed accessory already considered both client's comfort and user's convenience. AquaFlux probe is made of metal and slightly heavier compared to Epsilon. Therefore the black frame around the edge of the holder is suggested to be made of the magnet material to give AquaFlux more stability, firm insertion and it will result in an accurate reading.

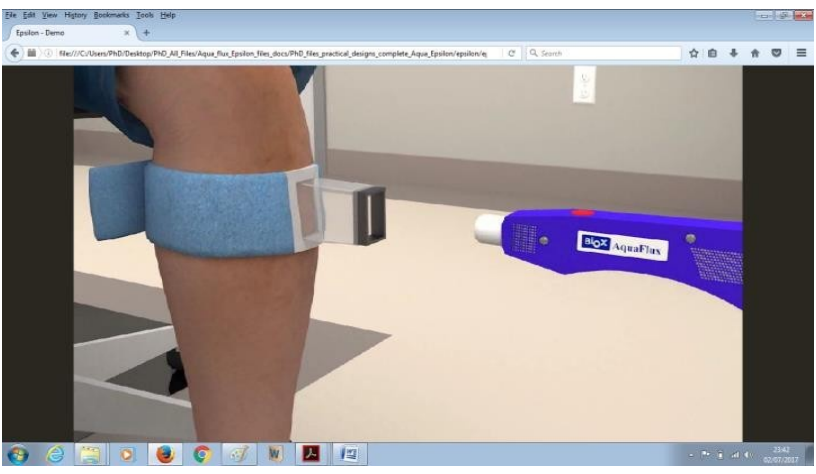

Fig. 17. AquaFlux new holder strap with magnet head 
After coming up with new ideas to utilise the work of AquaFlux and Epsilon, new hardwares have been added as accessorise with the instruments (holders). Several new design ideas evolved throughout the practical process of this research, some can be added immediately, and some are futuristic concepts and designs which help to enhance the process of conducting a skin measurement more efficiently and accurately. And, to serve a wide range of patients at the same time. The new upgraded design of AquaFlux enables the instrument to move in almost all directions to give more flexibility and ease of use for both clients and patients as shown in (Fig. 18) below:

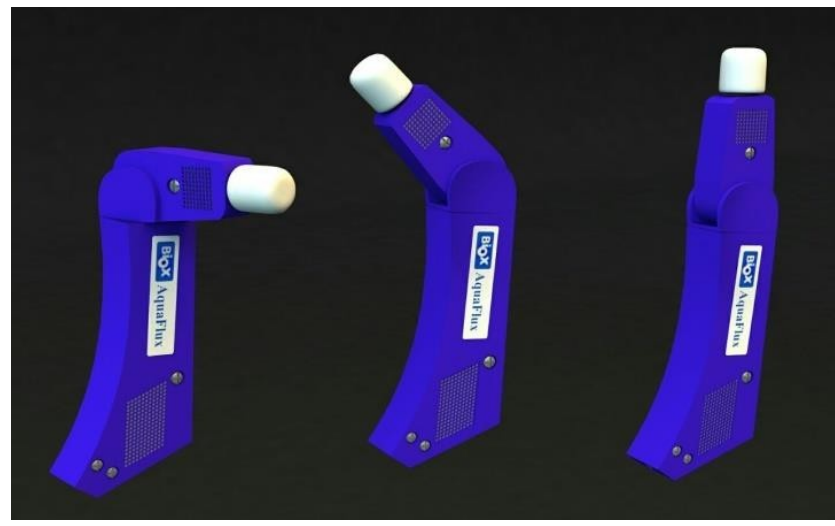

Fig. 18. Rotatable AquaFlux for more convenient use

The AquaFlux band is completely new concept design, very lightweight and it provides more comfort while the device is operational. It has padding that can be wrapped around different parts of the patient's body and it can be connected to PC or laptop via Bluetooth and save the inconvenience of having cables running around.

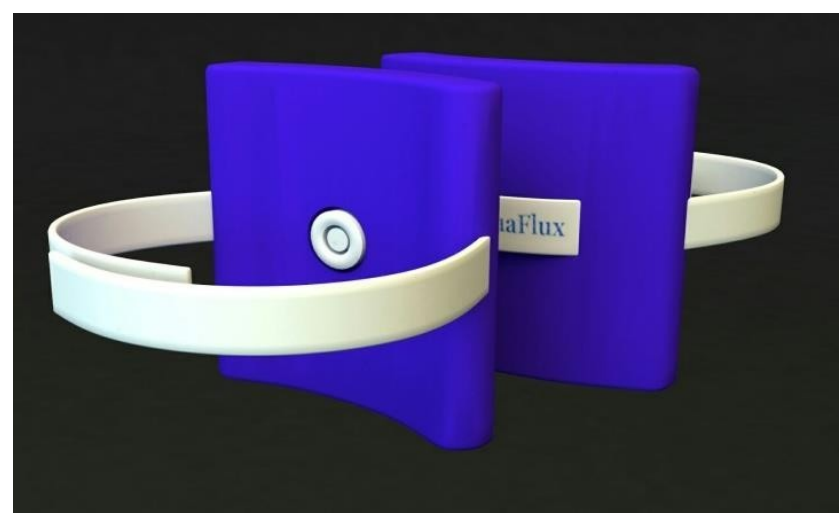

Fig. 19. AquaFlux band, new lightweight design with a strap

Epsilon moving head helps to conduct skin measurements in vertical/ horizontal positions. New screen added to the original design to provide accessibility and see results and images of human skin straight on the device. Functional buttons have been added to provide on probe operations such as On/Off, Scan, Reset. Epsilon can be completely portable.

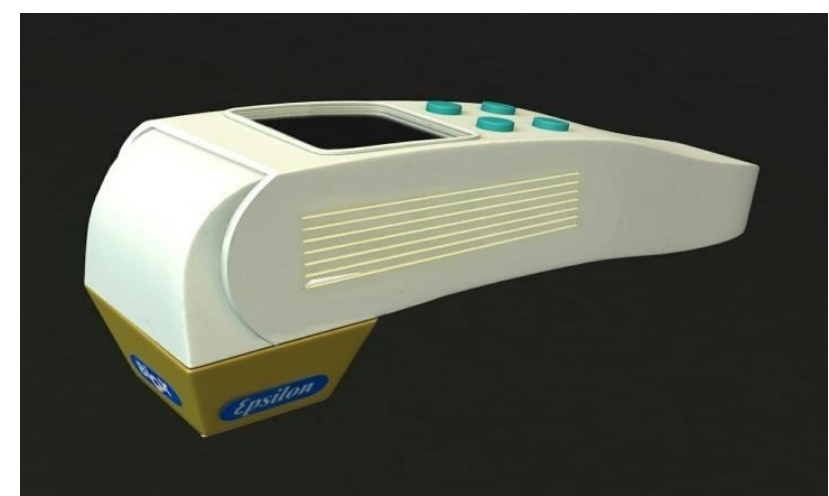

Fig. 20. Epsilon, moving head, screen and functional buttons

\section{CONCLUSION AND RECOMMENDATIONS}

The goal of this research paper was to present an overview of the 3D contents and using it in a more effective way than just viewing it or displaying it via a browser. And this is where the term VR (Virtual Reality) comes in, using the presented 3D content of the two skin treatment tools AquaFlux and Epsilon has enhanced the way these two instruments been working. With VR involved in the demonstration process and full interactivity at hand, the client or user can simply try and test these two medical instruments remotely using the internet as a platform and discover all their features and functions. In the virtual reality, we achieve a very immersive experience, which can come close to being at the location [10]. New hardware and accessories been presented too to show the power of marketing and conveying new ideas realistically to the rest of the world especially in the era of social media and internet were used on a huge scale. The completed work of designing an interactive virtual environment for AquaFlux and Epsilon could be easily upgraded and added to it many of today's latest trends in the world of Virtual Reality and technology, Augmented Reality, for instance, is a very competitive idea that this work could be upgraded to. VR (Virtual Reality) and AR (Augmented Reality) are unlocking a new level of interaction of the users with the specialised applications [11], not to mention 3D hologram, Holograms Technology gives tremendous interactive surface in collaborations' atmosphere. This technology is becoming an increasingly affordable medium as it inherited characteristics of a conventional workspace, a projected personal computer and touching capabilities. It creates an effective and efficient way of a presentation by offering an interactive display medium to cater the abilities of users' vision [12]. These two methods can show, demonstrate any designed object as if the user is laying his/her hand on it and discovering all its features and functionalities.

\section{REFERENCE S}

[1] Cellary, W. and Walczak, K. (2012). Interactive 3D Multimedia Content. London: Springer London.

[2] Paper, V., Virtualization Overview 1. , pp.1-11. 
[3] Anon, Instrument Development and Digital Signal Processing in Skin Measurements Zhang Xu School of Engineering London South Bank University Supervisors, Oct 2015.

[4] Installation, S. et al., 2013. Table of contents 1. , (November), pp.1-24.

[5] Ghanbarzadeh, R., Ghapanchi, A.H. \& Blumenstein, M., 2014. Application areas of multi-user virtual environments in the healthcare context. Studies in Health Technology and Informatics, 204, pp.38-46.

[6] Potkonjak, V., Gardner, M., Callaghan, V., Mattila, P., Guetl, C., Petrović, V. and Jovanović, K. (2016). Virtual laboratories for education in science, technology, and engineering: A review. Computers \& Education, 95, pp.309-327.

[7] Walczak, K., White, M. \& Cellary, W., 2004. Building Virtual and Augmented Reality Museum Exhibitions *. , 1(212), pp.135145.

[8] Yang, X. et al., 2008. Virtual Reality-Based Robotics Learning System., (September), pp.859-864.

[9] Shen, W. \& Zeng, W., 2011. Research of VR Modeling Technology Based on VRML and 3DS MAX. , pp.487-490.

[10] Naber, J., Krupitzer, C. and Becker, C. (2017). Transferring an Interactive Display Service to the Virtual Reality. Mannheim: IEEE, pp.1-4.

[11] Voinea, A., Moldoveanu, F. and Moldoveanu, A. (2017). 3D Model Generation of Human Musculoskel etal System Based on Image Processing. In: 21st International Conference on Control Systems and Computer Science. Bucharest: IEEE, pp.1-3.

[12] Wahab, N., Hasbullah, N., Ramli, S. and Zainuddin, N. (2016). Verification of A Battlefield Visualization Framework in Military Decision Making Using Holograms (3D) and Multi-Touch Technology. In: 2016 International Conference on Information and Communication Technology (ICICTM). Kuala Lumpur: IEEE, pp.1-2.

[13] Xiao, P. (2014). Biox Epsilon Model E100: Contact Imaging System. Available at: www.biox.biz/home/brochures.php 